\title{
A NEW QUANTITATIVE ETHNOECOLOGICAL APPROACH TO ASSESSING THE CONSERVATION STATUS OF PLANTS: A CASE STUDY OF DISTRICT TOR GHAR, PAKISTAN
}

\author{
SHAH, A. H. ${ }^{1 *}$ - MeHMOOd, A. ${ }^{2}-$ FAROOQ, M. ${ }^{1}-$ KHAN, K. R. ${ }^{1}-$ NAWAB, B. ${ }^{5}-$ SHAH, A. H. ${ }^{4}-$ \\ HUSSAIN, M. ${ }^{4}-$ ZOHRA, L. $^{5}-$ KHAN, S. M. ${ }^{3}-$ AHMAD, H. ${ }^{6}$ \\ ${ }^{1}$ Department of Botany, Govt. Post Graduate College, Mansehra, Pakistan \\ ${ }^{2}$ Department of Botany, Govt. Post Graduate College, Mandian, Pakistan \\ ${ }^{3}$ Department of Plant Sciences, Quaid-i-Azam University, Islamabad, Pakistan \\ ${ }^{4}$ Department of Botany, Hazara University, Mansehra, Pakistan \\ ${ }^{5}$ Department of Botany, GPGJC Saidu Sharif, Swat, Pakistan \\ ${ }^{6}$ Islamia College University, Peshawar, Pakistan \\ *Corresponding author \\ e-mail: abshah2086@gmail.com
}

(Received 28 ${ }^{\text {th }}$ Jan 2019; accepted $24^{\text {th }}$ May 2019)

\begin{abstract}
Ethnoecological knowledge refers to the local wisdom, innovations, traditional life style and practices of aboriginal communities. This knowledge also contributes a lot to understanding of conservation stata of culturally significant flora of a region. The present research work is the first one to focus on this particular aspect of cultural knowledge to assessing preliminary conservation status of 295 culturally significant plant species of district Tor Ghar. In such remote and less privileged areas interaction between plants and people is much stronger. Multistage random selection of 700 informants was made. The data was obtained through EPA, group discussion and semi structured interviews. The conservation status of all culturally significant plant species were assessed quantitatively by developing a new special mathematical relation that is $\mathrm{CS}=\mathrm{K}(\mathrm{OC}+\mathrm{AV}+\mathrm{CE}) / \mathrm{TF} \times \mathrm{RP}$. A scale was devised on the basis of CS value index and all 295 species were assigned to different IUCN categories. According to this classification 20 species were found critically endangered, 35 endangered, 56 vulnerable, 58 near threatened (rare) and 126 least concerned (secure). This categorization depicts a miniature of ethnoconservation of plants at local level. The current study leads to an enhanced understanding of cultural knowledge on mountain vegetation within the context of anthropogenic impacts and the role of indigenous communities in regional plant conservation strategies and future outlooks.
\end{abstract}

Keywords: local wisdom, aboriginal, endangered, threatened, ethno-conservation

\section{Introduction}

Biodiversity and culture of a region affect each other reciprocally. Local wisdom of communities popularly known as traditional knowledge, cultural knowledge, indigenous knowledge or local ecological knowledge. The body of knowledge usually includes understandings, beliefs, and practices that human societies develop longitudinally in relationship with their natural environment, and which are dynamic and co-evolving with social and ecological changes (Berkes, 2000; Zent, 2001; Von, 2011). This knowledge incorporates spiritual connections with the natural environment and the sustainable use of natural resources, and association between people, which are reflected in their language, narratives, social organisation, values, beliefs, and cultural 
laws and customs (Andrews, 2006). Every aspect of life is touched and covered by the culture of a particular region. According to Larid (1999) culture facilitates the association between humans and their environment. The perception of culture provides the basis of scientific disciplines such as ecological anthropology and scientific concepts such as bio-cultural diversity (Berkes et al., 2000).

According to Declaration of Belem (1988) there is an "inextricable link" between biological and cultural diversity. The term bio-cultural diversity was coined to describe the concept indicating the link (Posey, 1999). Human beings and their societies are an essential part of biodiversity, and according to their way of utilizing natural resources, they can be promoters of its sustainable use or drivers of its loss (Calvo, 2003). Wild biological resources are especially important for populations in rural areas, because these people depend directly on the extraction of local species to fulfill part of their daily requirements, such as wood, food, medicine, and timber (Boom, 1987; Prance et al., 1987; Phillips et al., 1994).

Participation of indigenous communities in decision making related to natural resource management has been regarded as a potential effort that can boost local wellbeing without harming biodiversity and ecosystem functions (United Nations, 1992; Schwartzman et al., 2000; Adams and Hutton, 2007; Berkes, 2007).

Local communities have more close association with biodiversity and their cultural practices and life styles influence biodiversity greatly. According to Convention on Biological Diversity, local communities showing a socially and geographically defined group of people may not homogeneous, living close to biodiversity and protected areas. They may have customary rights of use, distinctive knowledge and skills and direct impact on biodiversity as individuals or groups of individuals. These people are also described as having a close and unique relationship to their natural resources as a community. Locals communities may be dynamic in response to changing rural conditions, and therefore, may change their cultural practices and their perspectives on the importance of natural resources and biodiversity (Wiersum and Shackleton, 2005).

In order to protect and manage a particular ecological system, its cultural context is considered to be the most important one. Human relation to the natural environment has so for been studied mostly in biophysical terms; but there is now a growing acknowledgment that societies themselves have created detailed procedures to conserve and manage their resources. These methods are entrenched in their cultural values that have to be taken in to consideration if sustainable and reasonable human development is to become a truth (Bridgewater, 2002). These new trends have resulted in a change from a top down to a more community- based approach which aims to work closely with local people, depend on their cultural knowledge, and contribute to their livelihoods (Grimble and Laidlaw, 2002).

The convention on Biological Diversity (CBD) from 1992 stressed on parties to respect, preserve, and maintain knowledge, innovations and practices of indigenous and local communities having traditional lifestyles relevant for the conservation and sustainable use of biological diversity, and facilitate their wider involvement with the approval of bearers of such knowledge, innovations and practices, and encourage the reasonable sharing of the gains obtaining from the use of such knowledge, innovations and practices (McNeely, 2000).

Global environmental conservation policies and discourses have been increasingly influenced since 1980s, either verbally or practically, by the idea that conservation demands the coexistence of humans and nature (Adams et al., 2004; Wells and 
McShane, 2004). According to Cotton (1996) over long time, indigenous people have developed their own locality specific knowledge on plant use management and conservation. The United Nations Convention Environment and Development (UNCED, 1992) mentioned the important roles that indigenous populations play in both the conservation and sustainable use of natural resources while in turn maintaining biodiversity. Current use of cultural knowledge in conservation led to the novel idea of "ethnoconservation" in the late 1990s which is now well known conservation approach around the world (Rajeswar, 2001; Jules et al., 2008; Negi, 2010).

In a context where numerous conservation goals must be attained, local ecological knowledge can be a donor to a multidisciplinary conservation approach as well as foster transdisciplinary approaches when locals become partners and co-workers (Aswani, 2018). Assessment of conservation status cannot be absolute and needs periodic revision but taking various criteria at a time validates the conclusion for a considerable period of time or for a specific geographic locality (Domínguez Lozano et al., 2003; Broennimann et al., 2005).

In the present investigation local ecological knowledge has been incorporated in IUCN red list and used as a tool for assessing conservation status of culturally significant flora of district Tor Ghar with special reference to the following objectives.

1. To assess the existing threats to culturally significant plants in the study area

2. To evaluate how the cultural values of plant biodiversity contribute towards conservation.

3. To quantify the local wisdom regarding conservation status of the flora

\section{Materials and methods}

\section{Study area}

District Tor Ghar, a rigorous mountainous area of northern Pakistan located between $34^{\circ} 32^{\prime}$ and $34^{\circ} 50^{\prime} \mathrm{N}$, and $72^{\circ} 48^{\prime}$ and $72^{\circ} 58^{\prime} \mathrm{E}$ in Lesser Himalayas having strong cultural and traditional background besides rich plant biodiversity. The study area is inhabited by five well known tribes namely Basikhel, Akazai, Nusratkhel, Hassanzai and Medakhel (Fig. 1). Plants and peoples of the study area have close association and plants provide a number of societal benefits. Human culture is the main driver of plant biodiversity. Hence cultural knowledge was used as a tool for assessing conservation status of ethnospecies of the district.

\section{Data collection}

Villages of each tribal society as well as informants were selected randomly.700 informants shared their cultural knowledge related to conservation status through semi structured interviews, questionnaires, Ethnobotanical Participatory Appraisal (EPA) and group discussion.

\section{Field trips}

Extensive field trips of the study area were carried out during 2014 and 2015. Threatened and secure plant species were studied in their local habitats. Threatening factors and conservation efforts of indigenous tribes were recorded. Pressed, dried and poisoned specimens in triplicate deposited in herbarium of Hazara University, Mansehra, Pakistan. 


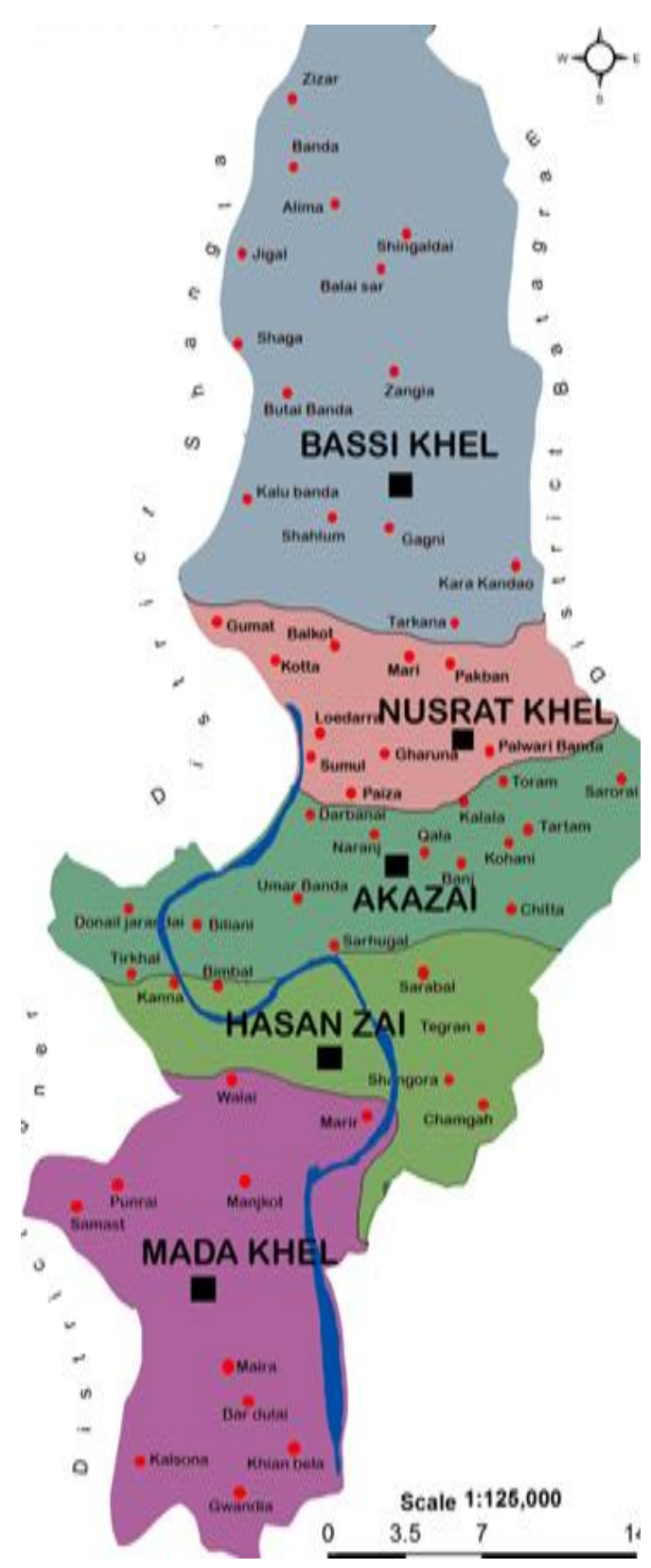

Figure 1. Map of district Tor Ghar showing different tribal belts

\section{Data analysis}

Recorded data from the questionnaires was shifted on Microsoft Excel sheet for further processing and evaluation. Whole conservation related data was analyzed successfully by developing a new simple mathematical relation named as CS equation.

\section{Development of a new equation}

A new mathematical equation was developed for assessment of conservation status, which is a novel approach in the field of ethnobiology for quantification of local wisdom. The idea was born during data collection and field visits that local wisdom if quantified properly to assessing conservation status of a plant species in a particular 
area, it would depict its conservation in a better way. During the study it has also been observed that locals adopted conservatory measures for protecting plant biodiversity. They protect the plants of their interest from fire and grazing and also cultivate useful plants. In this regard, a questionnaire was designed to get maximum information from the locals about conservation status of culturally significant flora of the area. Availability for the last ten years was recorded (increased, decreased or persistent). Occurrence of a plant species (abundance, moderate or rare) was noted. The most cited three important threatening factors of the study area (over exploitation, fire and grazing) were enlisted. Similarly, information regarding regeneration potential (high, moderate and low) was gathered. Data obtained from informants was cross checked by direct field observations during extensive field trips and analyzed by the following mathematical relation developed first time by the authors (Eq. 5).

As Conservation Status (CS) of a species is directly proportional to Occurrence (OC), Availability (AV) and Conservation Efforts (CE). This may be expressed mathematically as:

$$
\mathrm{CS} \alpha \mathrm{OC}+\mathrm{AV}+\mathrm{CE}
$$

Similarly Conservation Status (CS) is also directly proportional to Reproductive Potential (RP) of a species and can be represented as follows:

$$
\mathrm{CS} \alpha \mathrm{RP}
$$

where CS is inversely proportional to Threatening Factors (TF) shown mathematically as:

$$
\mathrm{CS} \alpha 1 / \mathrm{TF}
$$

By combining Equations 1, 2 and 3, the following equation obtained:

$$
\mathrm{CS} \alpha(\mathrm{OC}+\mathrm{AV}+\mathrm{CE}) / \mathrm{TF} \mathrm{X} \mathrm{RP}
$$

By substituting the sign of proportionality with constant $\mathrm{K}$, the final equation was thus obtained.

$$
\mathrm{CS}=\mathrm{K}(\mathrm{OC}+\mathrm{AV}+\mathrm{CE}) / \mathrm{TF} \mathrm{X} \mathrm{RP}
$$

where CS is the conservation status of a plant species, OC is occurrence, AV is availability for the last ten years, $\mathrm{CE}$ is conservation efforts by locals, TF is threatening factors and RP is regeneration potential of a plant species in a particular habitat.

The constant $\mathrm{K}$ was assigned a value 1 . Degree of occurrence was evaluated by giving values 3 for abundance, 2 for moderate and 1 for rare. Values for availability for the last ten years are 3 for increasing, 2 for persistent and 1 for decreasing. The most cited four conservation efforts by locals were considered in this equation and assigned a sum of value 4 to these ( 1 for each conservation effort). Conservation efforts are protection from grazing, cutting and fire. Cultivation of a plant species is also a conservation effort. Threatening factors considered in this formula on the basis of local perception were Over-exploitation, fire and overgrazing/overbrowsing. Each threatening 
factor was assigned a value 3 for extensive, 2 for moderate, 1 for low and 0 for absence of a threatening factor. Similarly, the values assigned to regeneration potential were 3 for high, 2 for moderate and 1 for low.

All 295 culturally significant plant species of the study area were assessed by the equation of CS in order to quantify the local wisdom. Keeping in view the CS index values, a scale was devised for assigning IUCN categories to the flora under investigation.

\section{2-scale for IUCN categories}

On the basis of $\mathrm{C} \mathrm{S}$ values index plant species were described according to IUCN classification as critically endangered, endangered, vulnerable, near threatened (rare) and least concerned (secure) (Table 1).

Table 1. Scale for IUCN categories

\begin{tabular}{c|c}
\hline Name of category & C S values \\
\hline Critically endangered & 0.1 to 1 \\
Endangered & 1.1 to 1.5 \\
Vulnerable & 1.6 to 2.0 \\
Rare & 2.1 to 2.5 \\
Least concerned (secure) & 2.6 and above \\
\hline
\end{tabular}

\section{Results}

Conservation status of 295 plant species was assessed tribewise and mean value was calculated for each recorded species (Table 2).

Table 2. Tribewise CS values and IUCN categorization

\begin{tabular}{|c|c|c|c|c|c|c|c|c|}
\hline \multirow{2}{*}{ Name of species } & \multirow{2}{*}{ Habit } & \multicolumn{7}{|c|}{ Tribe wise CS values } \\
\hline & & \begin{tabular}{|l|} 
Basikhel \\
\end{tabular} & Nusratkhel & Akazai & Hassanzai & Medakhel & Mean & Categories \\
\hline Podophyllum emodi Wall. ex Royle. & Herb & 0.5 & 0.5 & 0.5 & 0.5 & NR & 0.5 & $\mathrm{CE}$ \\
\hline Gentiana kurroo Royle. & Herb & 0.85 & 0.85 & NR & NR & & 0.85 & $\mathrm{CE}$ \\
\hline Dioscorea deltoidea Wall.exKunth. & Herb & 0.66 & 1.33 & 0.66 & 0.132 & 2 & 0.95 & $\mathrm{CE}$ \\
\hline Incarvellia emodi & Herb & & & 0.6 & & 1.32 & 0.96 & $\mathrm{CE}$ \\
\hline Arisaema utile Hook.f.ex. Schott. & Herb & 1 & & & & & 1 & $\mathrm{CE}$ \\
\hline Picris hieraciodes $\mathrm{L}$. & Herb & & & 1 & & & 1 & $\mathrm{CE}$ \\
\hline Citrullus colocynthis (Linn.) Schrad. & Herb & 1 & & & 1.32 & 1 & 1.1 & $E$ \\
\hline Asparagus officinalis $L$. & Herb & & & 1.3 & 1.5 & 1 & 1.2 & $\mathrm{E}$ \\
\hline Caralluma tuberculata N.E. Brown. & Herb & 1 & 1.5 & 1.3 & 1.32 & 1.5 & 1.32 & $\mathrm{E}$ \\
\hline Asparagus adscandens Roixb. & Herb & 0.5 & & & 1.5 & 2 & 1.33 & E \\
\hline $\begin{array}{l}\text { Astragalus graveolens Buch.- } \\
\text { Ham.exBenth. }\end{array}$ & Herb & 2 & 0 & 2 & & & 1.33 & $\mathrm{E}$ \\
\hline Colchicum luteum Baker. & Herb & 1 & 2 & 1 & & & 1.33 & $\mathrm{E}$ \\
\hline Allium griffithianum Boiss. & Herb & & & & 1.5 & 1.2 & 1.35 & $\mathrm{E}$ \\
\hline Arisaema flavum (Forssk.)Schott. & Herb & 1 & 2 & 1.33 & & & 1.44 & E \\
\hline Aerva sanguinolenta (Linn.) Blume. & Herb & & & & 1 & 2 & 1.5 & $\mathrm{E}$ \\
\hline Agave sisalana Perrine ex Engelm. & Herb & & & & 2 & 1 & 1.5 & $\mathrm{E}$ \\
\hline Crotolaria mediginea Lamk. & Herb & & & & & 1.5 & 1.5 & $\mathrm{E}$ \\
\hline Geranium lucidum L. & Herb & & & & & 1.5 & 1.5 & $\mathrm{E}$ \\
\hline Rumex vesicarius $\mathrm{L}$. & Herb & 1.5 & & & 1 & 2 & 1.5 & $\mathrm{E}$ \\
\hline
\end{tabular}


Shah et al.: A new quantitative ethnoecological approach to assessing the conservation status of plants: a case study of District Tor Ghar, Pakistan

- 10405 -

\begin{tabular}{|c|c|c|c|c|c|c|c|c|}
\hline \multirow{2}{*}{ Name of species } & \multirow{2}{*}{ Habit } & \multicolumn{7}{|c|}{ Tribe wise CS values } \\
\hline & & Basikhel & Nusratkhel & Akazai & Hassanzai & Medakhel & Mean & Categories \\
\hline $\begin{array}{l}\text { Geranium wallichianum D.Don ex } \\
\text { Sweet. }\end{array}$ & Herb & 1.6 & 1.6 & & 1.5 & & 1.56 & V \\
\hline Salvia lanata Roxburgh. & Herb & 1 & 1 & 1.5 & & 3 & 1.62 & $\mathrm{~V}$ \\
\hline Impatiens bicolor Royle. & Herb & 2 & 1.5 & & & & 1.75 & $\mathrm{~V}$ \\
\hline Bergenia ciliate Sternb. & Herb & 1.3 & 2 & 2 & & & 1.76 & $\mathrm{~V}$ \\
\hline Asparagus capitatus Baker. & Herb & 1 & 1.2 & & 2 & 3 & 1.8 & $\mathrm{~V}$ \\
\hline Polygonatum verticillatum & Herb & 2.6 & & 1 & & & 1.8 & $\mathrm{~V}$ \\
\hline Hypericum perforatum $\mathrm{L}$. & Herb & 1.6 & 2 & 2 & & & 1.86 & $\mathrm{~V}$ \\
\hline Salvia moorcroftiana Wall.ex Benth. & Herb & 2 & 2 & 2 & 1.5 & & 1.87 & $\mathrm{~V}$ \\
\hline Valeriana jatamansi Jones. & Herb & 1.5 & 1.2 & 2.4 & 2.6 & & 1.92 & $\mathrm{~V}$ \\
\hline Arthraxon prionodes (Steud.) Dandy. & Herb & & & & & 1.98 & 1.98 & $\mathrm{~V}$ \\
\hline $\begin{array}{c}\text { Alliaria petiolata (M.Bieb)Cavara\& } \\
\text { Grande. }\end{array}$ & Herb & 2 & 2 & & & & 2 & $\mathrm{~V}$ \\
\hline Arisaema jacquemontii Blume. & Herb & 2 & & & & & 2 & $\mathrm{~V}$ \\
\hline Cichorium intybu sL. & Herb & 2 & 2 & & & & 2 & $\mathrm{~V}$ \\
\hline $\begin{array}{l}\text { Codonopsis clematidea(Schrenk) } \\
\text { C.B.Clarke. }\end{array}$ & Herb & & & & & 2 & 2 & $\mathrm{~V}$ \\
\hline Convolvulus arvensis $\mathrm{L}$. & Herb & 2 & 2 & & & & 2 & $\mathrm{~V}$ \\
\hline Corchorus trilocularis $\mathrm{L}$. & Herb & & & & 2 & 2 & 2 & $\mathrm{~V}$ \\
\hline Erophila verna $\mathrm{L}$. & Herb & 2 & & & & & 2 & $\mathrm{~V}$ \\
\hline Euphorbia hispida Boiss. & Herb & & & & & 2 & 2 & $\mathrm{~V}$ \\
\hline Evolvulus alsinoides (L.) & Herb & & & & 2 & & 2 & $\mathrm{~V}$ \\
\hline Fumaria officinalis $\mathrm{L}$. & Herb & & & & & 2 & 2 & $\mathrm{~V}$ \\
\hline Mirabilis jalapa $\mathrm{L}$. & Herb & & & & & 2 & 2 & $\mathrm{~V}$ \\
\hline Poa bulbosa $\mathrm{L}$. & Herb & 2 & 2 & 2 & & & 2 & $\mathrm{~V}$ \\
\hline Polygonatum multiforum (L.) All. & Herb & 2 & 2 & & & & 2 & $\mathrm{~V}$ \\
\hline Swertia ciliata(G. Don) B.L. Burtt. & Herb & 2 & 2 & & & & 2 & $\mathrm{~V}$ \\
\hline Thymus linearis Benth. & Herb & 2 & & & & & 2 & $\mathrm{~V}$ \\
\hline Vicia hirsute (Linn.) S.F.Gray. & Herb & & & & & 2 & 2 & $\mathrm{~V}$ \\
\hline Viola odorata $\mathrm{L}$. & Herb & 2.4 & 1.6 & & & & 2 & $\mathrm{~V}$ \\
\hline $\begin{array}{l}\text { Bistorta amplexicaulis (D.Don) } \\
\text { Greene. }\end{array}$ & Herb & 3 & 0 & 2.6 & 2.6 & & 2 & $\mathrm{~V}$ \\
\hline Cissampelo spareira $\mathrm{L}$. & Herb & & & & 2.6 & 1.5 & 2 & $\mathrm{~V}$ \\
\hline Artemisia scoparia Waldst. \& Kit. & Herb & 2 & 2 & & 2 & 2.25 & 2 & $\mathrm{~V}$ \\
\hline Ajuga bracteosa Wall., Benth. & Herb & 2 & 1.6 & 2.6 & & & 2 & $\mathrm{~V}$ \\
\hline Tulipa clusiana(Hook.) Regel. & Herb & 2 & 2 & 2.4 & 2 & & 2.1 & $\mathrm{R}$ \\
\hline Taraxicum officinale Webb. & Herb & 2 & 2 & 2 & 2.25 & 2.4 & 2.13 & $\mathrm{R}$ \\
\hline Aristida depressa Retz. & Herb & 2.14 & 2.14 & 2.14 & & & 2.14 & $\mathrm{R}$ \\
\hline Narcissus tazetta $\mathrm{L}$. & Herb & 3 & 1.5 & & & 2 & 2.16 & $\mathrm{R}$ \\
\hline $\begin{array}{l}\text { Astragalus amherstianus Royle ex } \\
\text { Benth. }\end{array}$ & Herb & 2 & 2 & 2.6 & & & 2.2 & $\mathrm{R}$ \\
\hline Calendula arvensis $\mathrm{L}$. & Herb & 2 & 2 & 2.6 & & & 2.2 & $\mathrm{R}$ \\
\hline Cardamine hirsute $\mathrm{L}$. & Herb & 2 & 2 & & & 2.6 & 2.2 & $\mathrm{R}$ \\
\hline Clematis Montana Buch. & Herb & 2 & 2.6 & 2 & & & 2.2 & $\mathrm{R}$ \\
\hline Dicliptera bupleuroides Nees. & Herb & 2.6 & 2 & 2 & & & 2.2 & $\mathrm{R}$ \\
\hline Lepidium aucheri Boiss. & Herb & 2 & 2 & & 2.6 & & 2.2 & $\mathrm{R}$ \\
\hline Silene vulgaris (Moench) Garcke. & Herb & 2.4 & & & 2 & & 2.2 & $\mathrm{R}$ \\
\hline Vernonia Sinerea (L.)Lees. & Herb & 2.4 & & 2 & & & 2.2 & $\mathrm{R}$ \\
\hline Brachiaria ramosa(Linn.) Stap. & Herb & & & & 1.98 & 2.49 & 2.23 & $\mathrm{R}$ \\
\hline Bothriochloa ischaemum (L.) Keng. & Herb & & & & & 2.25 & 2.25 & $\mathrm{R}$ \\
\hline Commelina benghalensis $\mathrm{L}$. & Herb & 2.5 & 2.5 & 2 & 2 & & 2.25 & $\mathrm{R}$ \\
\hline Apluda aristata $\mathrm{L}$. & Herb & 2.14 & 2.14 & 2.14 & 2.5 & 2.49 & 2.28 & $\mathrm{R}$ \\
\hline Commelina poludosa Blume. & Herb & 1.6 & & & 3 & & 2.3 & $\mathrm{R}$ \\
\hline
\end{tabular}


Shah et al.: A new quantitative ethnoecological approach to assessing the conservation status of plants: a case study of District Tor Ghar, Pakistan

- 10406 -

\begin{tabular}{|c|c|c|c|c|c|c|c|c|}
\hline \multirow{2}{*}{ Name of species } & \multirow{2}{*}{ Habit } & \multicolumn{7}{|c|}{ Tribe wise CS values } \\
\hline & & Basikhel & Nusratkhel & Akazai & Hassanzai & Medakhel & Mean & Categories \\
\hline Cynoglossum lanceolatum Forssk. & Herb & 2.6 & 2 & & & & 2.3 & $\mathrm{R}$ \\
\hline Senesio chrysanthemoides DC. & Herb & 2 & 2.6 & & & & 2.3 & $\mathrm{R}$ \\
\hline Verbena officinalis $\mathrm{L}$. & Herb & & & & 2 & 2.6 & 2.3 & $\mathrm{R}$ \\
\hline Sisymbrium irrio L. & Herb & 1.6 & 1.6 & 2.6 & 2.8 & 3 & 2.32 & $\mathrm{R}$ \\
\hline Artemisia absinthium $\mathrm{L}$. & Herb & 2 & 2 & 3 & & & 2.33 & $\mathrm{R}$ \\
\hline Achillea millifolium $\mathrm{L}$. & Herb & 2.6 & 2.6 & 2 & & & 2.4 & $\mathrm{R}$ \\
\hline Achyranthus aspera $\mathrm{L}$. & Herb & 3 & 2 & 2 & 2.6 & & 2.4 & $\mathrm{R}$ \\
\hline Acorus calamus $\mathrm{L}$. & Herb & 2 & 2.6 & & 2.6 & & 2.4 & $\mathrm{R}$ \\
\hline Tragus roxburghii Panigrahi. & Herb & & & & & 2.4 & 2.4 & $\mathrm{R}$ \\
\hline Stellaria media (L.) Vill. & Herb & 2.4 & & & 2.25 & 2.6 & 2.41 & $\mathrm{R}$ \\
\hline Calamagrostis decora Hook. f., Fl. Bri. & Herb & 2.4 & 2.4 & 2.4 & 2.5 & 2.4 & 2.42 & $\mathrm{R}$ \\
\hline Deschampsiacae spitosa $\mathrm{L}$. & Herb & 2.4 & 2.4 & 2.4 & 2.5 & 2.4 & 2.42 & $\mathrm{R}$ \\
\hline Trifolium pratense $\mathrm{L}$. & Herb & 2.5 & & & 2.4 & 2.4 & 2.43 & $\mathrm{R}$ \\
\hline Datura stramonium $\mathrm{L}$. & Herb & 2.6 & 2 & 2 & 2.6 & 3 & 2.44 & $\mathrm{R}$ \\
\hline Imperata cylindrical (L)P. Beauv. & Herb & 2.66 & & 2.4 & 2.4 & & 2.48 & $\mathrm{R}$ \\
\hline Dactylotenium aegyptium(L) P.Beauv. & Herb & 2.4 & 2.4 & 2.4 & 3 & 2.25 & 2.49 & $\mathrm{R}$ \\
\hline Chrysopogon serrulatusTrin. & Herb & & & & & 2.49 & 2.49 & $\mathrm{R}$ \\
\hline Eragrostis ciliaris $(\mathrm{L}.) \mathrm{R} . \mathrm{Br}$. & Herb & & & & & 2.49 & 2.49 & $\mathrm{R}$ \\
\hline Agrostis stolonifera $\mathrm{L}$. & Herb & 2.5 & 2.5 & 2.5 & & 2.49 & 2.49 & $\mathrm{R}$ \\
\hline Euphorbia wallichii $\mathrm{Hk}$. & Herb & 2.5 & 2.5 & & & & 2.5 & $\mathrm{R}$ \\
\hline Impatiens edgeworthii $\mathrm{Hk} . \mathrm{F}$. & Herb & 3 & 2 & & & & 2.5 & $\mathrm{R}$ \\
\hline Phragmites australis(Cay.) Trin. & Herb & & & & 2.4 & 2.6 & 2.5 & $\mathrm{R}$ \\
\hline Colocasia esculenta (Linn.) Schott. & Herb & 2.6 & 3 & & 1.5 & 3 & 2.5 & $\mathrm{R}$ \\
\hline Ricinus communis L. & Herb & 3 & 2 & & 2.6 & 2.6 & 2.5 & $\mathrm{R}$ \\
\hline CarthmusoxycanthaM.Bieb. & Herb & 2 & 2 & 2.6 & 3.32 & 3 & 2.58 & $S$ \\
\hline Avena fetua $\mathrm{L}$. & Herb & 3 & & 2.4 & 1.98 & 3 & 2.59 & $S$ \\
\hline Achyranthes bidentata Blume. & Herb & 2.6 & 2.6 & & & & 2.6 & $\mathrm{~S}$ \\
\hline Amaranthus spinesus $\mathrm{L}$. & Herb & 3 & 2 & & 2.4 & 3 & 2.6 & $S$ \\
\hline Borreria articularis (L.F.) FN . Will. & Herb & & & & & 2.6 & 2.6 & $S$ \\
\hline Caltha alba Camb. & Herb & 2.6 & & & & & 2.6 & $\mathrm{~S}$ \\
\hline Campanula benthamii Wall. & Herb & & & & & 2.6 & 2.6 & $S$ \\
\hline Cardiospermum halicacabum $\mathrm{L}$. & Herb & 2.6 & & & 2.6 & 2.6 & 2.6 & $S$ \\
\hline Centaurea iberica Trevir\&Spreng. & Herb & 2.6 & & & & & 2.6 & $\mathrm{~S}$ \\
\hline Gagea lutea $(\mathrm{L})$ Ker-Gawl. & Herb & 2.6 & & & & & 2.6 & $S$ \\
\hline Galium aparine $\mathrm{L}$. & Herb & 2.6 & & & & & 2.6 & $S$ \\
\hline Lactuca serriola $\mathrm{L}$. & Herb & 2.6 & & & & & 2.6 & $S$ \\
\hline Oenthera rosea $\mathrm{L}$. & Herb & & & & & 2.6 & 2.6 & $S$ \\
\hline Onosma hispidaWall. Ex G. Don. & Herb & 2.6 & 2.6 & & & & 2.6 & $S$ \\
\hline Persicaria hydropiper (L.) Spach. & Herb & & & & & 2.6 & 2.6 & $S$ \\
\hline Polygonum aviculare $\mathrm{L}$. & Herb & 2.6 & 2.6 & 2.6 & & & 2.6 & $\mathrm{~S}$ \\
\hline Polygonum plebejum R. Br. & Herb & & & & & 2.6 & 2.6 & $\mathrm{~S}$ \\
\hline Stachys parviflora Benth. & Herb & 2.6 & 2.6 & & 2.6 & 2.6 & 2.6 & $S$ \\
\hline Torilislepto phyla (L.) Reichb. & Herb & & & & & 2.6 & 2.6 & $\mathrm{~S}$ \\
\hline Sorghum haleeparse (L) Pers. & Herb & 2.66 & & 2.4 & 3 & 2.4 & 2.61 & $\mathrm{~S}$ \\
\hline Phalaris minor Retz. & Herb & 3 & & 2.5 & 2.5 & 2.49 & 2.62 & $S$ \\
\hline Fumaria indica(Hausskn) Pusley. & Herb & 2.5 & 2.5 & & 2.6 & 3 & 2.65 & $\mathrm{~S}$ \\
\hline Aethusa cynapium $\mathrm{L}$. & Herb & 2.66 & & & & & 2.66 & $\mathrm{~S}$ \\
\hline Bupleurum falcatum $\mathrm{L}$. & Herb & 2.66 & & & & & 2.66 & $S$ \\
\hline Nepeta cataria $\mathrm{L}$. & Herb & 2.66 & & & & & 2.66 & $\mathrm{~S}$ \\
\hline Dichanthium annulatum(Forssk) Stapf. & Herb & 3 & & 3 & 2.15 & 2.5 & 2.66 & $\mathrm{~S}$ \\
\hline Artemisia vulgaris $\mathrm{L}$. & Herb & 2.5 & 2.5 & & & 3 & 2.66 & $\mathrm{~S}$ \\
\hline
\end{tabular}


Shah et al.: A new quantitative ethnoecological approach to assessing the conservation status of plants: a case study of District Tor Ghar, Pakistan

- 10407 -

\begin{tabular}{|c|c|c|c|c|c|c|c|c|}
\hline \multirow{2}{*}{ Name of species } & \multirow{2}{*}{ Habit } & \multicolumn{7}{|c|}{ Tribe wise CS values } \\
\hline & & Basikhel & Nusratkhel & Akazai & Hassanzai & Medakhel & Mean & Categories \\
\hline Cuscuta gigantea Griff. & Herb & 3 & & & 3 & 2 & 2.66 & $\mathrm{~S}$ \\
\hline Mentha spicata $\mathrm{L}$. & Herb & 3 & 3 & & & 2 & 2.66 & $S$ \\
\hline Pulicaria crispa(Forssk.) Oliv. & Herb & 2 & & & 3 & 3 & 2.66 & $S$ \\
\hline Silene conidia $\mathrm{L}$. & Herb & 3 & 3 & & 2 & & 2.66 & $\mathrm{~S}$ \\
\hline Digitaria nodosa Perl. & Herb & 2.5 & 3 & 3 & 2.5 & 2.49 & 2.69 & $S$ \\
\hline Cynodon dactylon $(\mathrm{L})$ Pers. & Herb & 2.5 & 2.5 & 3 & 2.5 & 3 & 2.7 & $S$ \\
\hline Viola canescensWall. ex Roxb. & Herb & 3 & & 2.4 & & & 2.7 & $\mathrm{~S}$ \\
\hline Rumex hastatusD. Don. Prodr. & Herb & 2.4 & 3 & 3 & 2.4 & 3 & 2.76 & $S$ \\
\hline Medicago polymorpha $\mathrm{L}$. & Herb & 3 & 3 & 3 & 2.5 & 2.4 & 2.78 & $S$ \\
\hline Clematis grata Wall. & Herb & 2.6 & & & & 3 & 2.8 & $\mathrm{~S}$ \\
\hline $\begin{array}{c}\text { Fragaria nubicola(Hook.f.) Lindl. ex } \\
\text { Lacaita. }\end{array}$ & Herb & 3 & & 3 & 2.4 & & 2.8 & $S$ \\
\hline Chenopodium murale $\mathrm{L}$. & Herb & 3 & & & 3 & 2.6 & 2.86 & $S$ \\
\hline Rumex acetosa $\mathrm{L}$. & Herb & 2.6 & 3 & 3 & & & 2.86 & $S$ \\
\hline Solanum incanum L. & Herb & 3 & & & 2.6 & 3 & 2.86 & $\mathrm{~S}$ \\
\hline Chenopodium album $\mathrm{L}$. & Herb & 2.4 & 2.4 & 3 & & 3.75 & 2.88 & $\mathrm{~S}$ \\
\hline Desmostachya bipinnata $(\mathrm{L})$ Stapf. & Herb & 3 & 3 & 3 & 3 & 2.49 & 2.89 & $\mathrm{~S}$ \\
\hline Rumex dentatus $\mathrm{L}$. & Herb & 3 & 3 & 3 & & 2.6 & 2.9 & $\mathrm{~S}$ \\
\hline Ajuga reptan $\mathrm{L}$. & Herb & 3 & & & & & 3 & $S$ \\
\hline Alloteropsis cimicina (L.) Stapf. & Herb & & & & & 3 & 3 & $S$ \\
\hline $\begin{array}{l}\text { Asplenium septentrionale(Linnaeus) } \\
\text { Hoffmann. }\end{array}$ & Herb & 4 & 2 & & & & 3 & $S$ \\
\hline Canna indica $\mathrm{L}$. & Herb & & & & & 3 & 3 & $S$ \\
\hline Chenopodium botrys $\mathrm{L}$. & Herb & 3 & & & & 3 & 3 & $\mathrm{~S}$ \\
\hline Cleome scaposa DC. Prodr. & Herb & & & & & 3 & 3 & $\mathrm{~S}$ \\
\hline Coronopus didymus (L.) Sm. & Herb & 2 & 2 & 5 & & 3 & 3 & $S$ \\
\hline Euphorbia hirta $\mathrm{L}$. & Herb & 4 & & & 2 & 3 & 3 & $S$ \\
\hline Euphorbia prostrate Aiton. & Herb & & & & & 3 & 3 & $S$ \\
\hline Lathyrus aphaca $\mathrm{L}$. & Herb & 3 & 3 & 3 & 3 & & 3 & $S$ \\
\hline Leptochloa panicea(Retz.) Ohwi & Herb & & & & & 3 & 3 & $\mathrm{~S}$ \\
\hline Malva neglecta Wall. & Herb & 3 & 3 & 3 & 3 & 3 & 3 & $\mathrm{~S}$ \\
\hline Malva sylvestris $\mathrm{L}$. & Herb & 3 & & & 3 & 3 & 3 & $\mathrm{~S}$ \\
\hline Marrubium vulgare $\mathrm{L}$. & Herb & 4 & & 2 & & & 3 & $S$ \\
\hline Melilotus officinalis (L.)Desr. & Herb & 3 & 3 & 3 & & & 3 & $S$ \\
\hline Oxalis carniculatus $\mathrm{L}$. & Herb & 3 & 3 & 3 & 3 & 3 & 3 & $\mathrm{~S}$ \\
\hline Plantago major $\mathrm{L}$. & Herb & 3 & 3 & & & & 3 & $S$ \\
\hline Trifolium repens $\mathrm{L}$. & Herb & 3 & 3 & 3 & 3 & 3 & 3 & $\mathrm{~S}$ \\
\hline Verbascum thapsus $\mathrm{L}$. & Herb & 3 & & & 4 & 2 & 3 & $\mathrm{~S}$ \\
\hline Trichodesma indicum (L.) R. Br. Prodr. & Herb & 2.6 & & & 3.9 & 2.6 & 3 & $S$ \\
\hline Sonchus asper $(\mathrm{L})$ Hill. & Herb & 3 & 2 & 3.6 & 3 & 3.75 & 3 & $\mathrm{~S}$ \\
\hline Aerva javanica (Burm.f) Juss. & Herb & 2 & 4 & 4 & 2 & 3.75 & 3.15 & $S$ \\
\hline Amaranthus caudatus L. & Herb & 3 & 3.75 & 3 & 3 & 3 & 3.15 & $S$ \\
\hline Duchesnea indica(Andr.)Focke. & Herb & 3.75 & 2.5 & 3 & 3 & 3.75 & 3.2 & $S$ \\
\hline Plantago lanceolata $\mathrm{L}$. & Herb & 3 & & 3 & 3.9 & 3 & 3.2 & $\mathrm{~S}$ \\
\hline Amaranthus viridis $\mathrm{L}$. & Herb & 3.5 & 3 & & & & 3.25 & $S$ \\
\hline Chamaemelum nobile(L.) All. & Herb & 3.3 & & & & & 3.3 & $S$ \\
\hline Cuscuta reflexa Roxb. & Herb & 2.6 & 2.6 & & 4 & 4 & 3.3 & $\mathrm{~S}$ \\
\hline Equisetum ramosissimum Desf. & Herb & 3 & & & 3 & 4 & 3.33 & $S$ \\
\hline Solanum nigrum $\mathrm{L}$. & Herb & 2.5 & 3 & 3.75 & 3.75 & 3.75 & 3.35 & $\mathrm{~S}$ \\
\hline Nasturtium officinale $\mathrm{R}$. Br. & Herb & 3.75 & 3 & 5 & 3 & 2.25 & 3.4 & $S$ \\
\hline Conyza canadensis (L.) Corgn. & Herb & 3.75 & 3.75 & & 3.75 & 2.5 & 3.43 & $\mathrm{~S}$ \\
\hline Arundo donax $\mathrm{L}$. & Herb & 3 & 3.75 & 3.75 & 3.75 & 3 & 3.45 & $\mathrm{~S}$ \\
\hline
\end{tabular}


Shah et al.: A new quantitative ethnoecological approach to assessing the conservation status of plants: a case study of District Tor Ghar, Pakistan

- 10408 -

\begin{tabular}{|c|c|c|c|c|c|c|c|c|}
\hline \multirow{2}{*}{ Name of species } & \multirow{2}{*}{ Habit } & \multicolumn{7}{|c|}{ Tribe wise CS values } \\
\hline & & \begin{tabular}{|l|} 
Basikhel \\
\end{tabular} & Nusratkhel & Akazai & Hassanzai & Medakhel & Mean & Categories \\
\hline Neslia apiculata Fisch. & Herb & & & & 3.9 & 3 & 3.45 & $\mathrm{~S}$ \\
\hline Capsella bursa-pestoris L. & Herb & 2.6 & 2.5 & & 3.75 & 4.98 & 3.45 & $S$ \\
\hline Cyperus cyperoides $\mathrm{L}$. & Herb & 2.5 & 2.5 & & 4.98 & 4 & 3.49 & $S$ \\
\hline Anisomeles indica(L.) O. Kuntze. & Herb & 4 & & 3 & & & 3.5 & $\mathrm{~S}$ \\
\hline Urtica dioica $\mathrm{L}$. & Herb & 3 & & 4 & & & 3.5 & $\mathrm{~S}$ \\
\hline Chenopodium ambrosioides $\mathrm{L}$. & Herb & 3 & & & 3.9 & 4 & 3.63 & $S$ \\
\hline Aloe vera $(\mathrm{L}$.$) Burm.$ & Herb & 3 & 4 & & 4 & 4 & 3.75 & $\mathrm{~S}$ \\
\hline Cannabis sativa $\mathrm{L}$. & Herb & 4 & & 4 & 3.75 & 4 & 3.93 & $S$ \\
\hline Silybum marianum $(\mathrm{L})$ Gaertn. & Herb & 5 & 2.6 & 3.9 & 4.98 & 3.32 & 3.96 & $\mathrm{~S}$ \\
\hline Adiantum incisum Forssk. & Herb & 4 & & & & & 4 & $\mathrm{~S}$ \\
\hline Galinsoga parviflora Cavanilles. & Herb & & & & 4 & & 4 & $S$ \\
\hline Portulaca oleracea $\mathrm{L}$. & Herb & 4 & & & & & 4 & $S$ \\
\hline Pteris cretica $\mathrm{L}$. & Herb & & & & 4 & & 4 & $\mathrm{~S}$ \\
\hline Ranunculus arvensis $\mathrm{L}$. & Herb & 4 & & 4 & & & 4 & $\mathrm{~S}$ \\
\hline Ranunculus scleratus $\mathrm{L}$. & Herb & 4 & & & & 4 & 4 & $\mathrm{~S}$ \\
\hline Solena amplexicaulis(Lam.)Gandhi. & Herb & 4 & & & & & 4 & $\mathrm{~S}$ \\
\hline $\begin{array}{l}\text { Boenninghausenia albiflora(Hook.) } \\
\text { Reichb. } \\
\end{array}$ & Herb & 4 & & & & & 4 & $\mathrm{~S}$ \\
\hline Foeniculum vulgare Mill. & Herb & 4.5 & 4 & & 3.75 & & 4 & $S$ \\
\hline Adiantum capillus- veneris $\mathrm{L}$. & Herb & 5 & 4 & & 4 & & 4.33 & $S$ \\
\hline Xanthium strumarium $\mathrm{L}$. & Herb & 5 & 5 & 3.3 & & & 4.43 & $S$ \\
\hline Mentha longifolia $(\mathrm{L}$.$) Huds.$ & Herb & 5 & 4 & 4 & & 4.98 & 4.49 & $S$ \\
\hline Mentha arvensis $\mathrm{L}$. & Herb & 5 & 5 & 5 & 4 & & 4.75 & $S$ \\
\hline Adiantum venustum $\mathrm{D}$. Don. & Herb & & & & 5 & & 5 & $S$ \\
\hline Eryngium Sp. L. & Herb & 5 & & & & & 5 & $S$ \\
\hline Polystichum lonchitis $\mathrm{L}$. & Herb & 5 & & & & & 5 & $S$ \\
\hline Ranunculus muricatus $\mathrm{L}$. & Herb & 5 & 5 & & & & 5 & $S$ \\
\hline Euphorbia helioscopia L. & Herb & 7.5 & & & 4.98 & 4.98 & 5.82 & $\mathrm{~S}$ \\
\hline $\begin{array}{l}\text { Skimmia laureola(DC.) Sieb. \&Zucc. } \\
\text { ex Walp. }\end{array}$ & Shrub & 0.5 & 0.4 & 0.5 & 0.5 & 0.5 & 0.48 & $\mathrm{CE}$ \\
\hline Woodfordia fruticosa(L.)S.Kurz. & Shrub & 1 & 0.33 & 0.4 & 1 & 1.2 & 0.78 & $\mathrm{CE}$ \\
\hline Periploca aphylla Dcne. & Shrub & 1 & 0.66 & 1 & & & 0.88 & $\mathrm{CE}$ \\
\hline Opuntia dillenii Haw. & Shrub & 1 & 0.75 & 1 & 1 & 1.32 & 1 & $\mathrm{CE}$ \\
\hline Hedra nepalensis K.Koch. & Shrub & 0.5 & 1 & 1.5 & & & 1 & $\mathrm{CE}$ \\
\hline $\begin{array}{l}\text { Nannorrhops ritchieana (Griff.) } \\
\text { Aitchison. } \\
\end{array}$ & Shrub & & & & 1 & 1 & 1 & $\mathrm{CE}$ \\
\hline Vitis jacquemontii Parker. & Shrub & & 1 & & & & 1 & $\mathrm{CE}$ \\
\hline Viburnum grandiflorumWall. ex DC. & Shrub & 0.6 & & 1.5 & & & 1 & $\mathrm{CE}$ \\
\hline Viburnum cotinifolium D. Don. & Shrub & 1.2 & 1.2 & & 1 & & 1.13 & $\mathrm{E}$ \\
\hline Buxus wallichiana Bill. & Shrub & 1 & & 1.3 & & & 1.15 & $\mathrm{E}$ \\
\hline Buddleja crispa Bth. & Shrub & 1 & 1 & 2 & 1.5 & 1.5 & 1.4 & $\mathrm{E}$ \\
\hline Vitis parvifolia Roxb. & Shrub & & & 1.5 & & & 1.5 & $\mathrm{E}$ \\
\hline Carissa opaca Stapf. en Haines. & Shrub & 1.5 & 2 & 1 & 1.2 & 2 & 1.54 & $\mathrm{~V}$ \\
\hline Hypericum oblongifolium $\mathrm{L}$. & Shrub & 2 & & 1 & 1.2 & 2 & 1.55 & $\mathrm{~V}$ \\
\hline Colebrookia oppositifolia Smith. & Shrub & 0.75 & 0.75 & 1 & 2.6 & 3.32 & 1.68 & $\mathrm{~V}$ \\
\hline Cotoneaster bacillarisWall. ex Lindl. & Shrub & 1.5 & & 2 & & & 1.75 & $\mathrm{~V}$ \\
\hline Justicia adhatoda L. & Shrub & 2 & 1.5 & 2 & 2 & 1.5 & 1.8 & $\mathrm{~V}$ \\
\hline Caesalpinia decapitala(Roth) Alston. & Shrub & 1.5 & & 2 & 1.5 & 2.4 & 1.85 & $\mathrm{~V}$ \\
\hline Cotoneaster nummularia Fish \&Mey. & Shrub & 1.2 & & 2 & 2.4 & & 1.86 & $\mathrm{~V}$ \\
\hline Bambusa glaucescens(Willd.) Sieb. & Shrub & 1 & & & 3 & & 2 & $\mathrm{~V}$ \\
\hline Daphne mисronata Royle. & Shrub & 2 & & & 2 & 2 & 2 & $\mathrm{~V}$ \\
\hline Jasminum nudiflorum Lindl. & Shrub & 2 & 2 & & & & 2 & $\mathrm{~V}$ \\
\hline
\end{tabular}


Shah et al.: A new quantitative ethnoecological approach to assessing the conservation status of plants: a case study of District Tor Ghar, Pakistan

- 10409 -

\begin{tabular}{|c|c|c|c|c|c|c|c|c|}
\hline \multirow{2}{*}{ Name of species } & \multirow{2}{*}{ Habit } & \multicolumn{7}{|c|}{ Tribe wise CS values } \\
\hline & & Basikhel & Nusratkhel & Akazai & Hassanzai & Medakhel & Mean & Categories \\
\hline Otostegia limbata (Bth) Boiss. & Shrub & 2 & & 2 & 2 & 2 & 2 & $\mathrm{~V}$ \\
\hline Yucca aloifolia $\mathrm{L}$. & Shrub & & & & 2 & 2 & 2 & $\mathrm{~V}$ \\
\hline Ziziphus oxyphylla Edgew. & Shrub & 2 & & & & & 2 & $\mathrm{~V}$ \\
\hline $\begin{array}{l}\text { Maytenus royleanus(Wall. ex Lawson) } \\
\text { Cufodontis. }\end{array}$ & Shrub & 2 & 2 & 1.6 & 2.6 & 2 & 2 & $\mathrm{~V}$ \\
\hline Berberis lycium Royle. & Shrub & 2.5 & 2.1 & 2.4 & 1 & 2.49 & 2 & $\mathrm{~V}$ \\
\hline $\begin{array}{l}\text { Debregeasia salicifolia(D.Don) } \\
\text { Rendle. } \\
\end{array}$ & Shrub & 2.4 & 1.6 & 2.4 & & & 2.1 & $\mathrm{R}$ \\
\hline Isodon rugosus(Wall. ex Benth.) Codd. & Shrub & 2 & 2 & 2.6 & & 2 & 2.15 & $\mathrm{R}$ \\
\hline Sarcococca saligna (D.Don) Muell. & Shrub & 2 & 3 & 1.5 & & & 2.16 & $\mathrm{R}$ \\
\hline Nerium oleander L. & Shrub & 2.6 & 2 & & & 2 & 2.2 & $\mathrm{R}$ \\
\hline Rubus fructicosusHook .f. & Shrub & 2 & 2.5 & 2.4 & & 2 & 2.25 & $\mathrm{R}$ \\
\hline $\begin{array}{l}\text { Andrachne cordifolia(Wall. ex Decne.) } \\
\text { Muell. }\end{array}$ & Shrub & 2.4 & 2.4 & 2 & 2.6 & 2 & 2.28 & $\mathrm{R}$ \\
\hline Jasminum humile L. & Shrub & 2 & & 2 & 3 & & 2.33 & $\mathrm{R}$ \\
\hline Vitex negundo L. & Shrub & 2 & 2.6 & 2.5 & 2 & 2.6 & 2.34 & $\mathrm{R}$ \\
\hline Zanthoxylum armatum DC. & Shrub & 2 & 2.6 & 3 & 2 & 2.4 & 2.4 & $\mathrm{R}$ \\
\hline Cotinus coggyria Scop. & Shrub & 2.4 & 2.4 & 2.6 & 2.4 & & 2.45 & $\mathrm{R}$ \\
\hline Vitis vinifera $\mathrm{L}$. & Shrub & 2.6 & & & & & 2.6 & $S$ \\
\hline Myrsine Africana L. & Shrub & 3 & 3 & 3 & 2.4 & 2.4 & 2.76 & $\mathrm{~S}$ \\
\hline Indigofera heteranthaWall.ex rand. & Shrub & 2.5 & 2.5 & 3 & 2.5 & 3.6 & 2.82 & $\mathrm{~S}$ \\
\hline Calotropis procera(Ait.) Ait. F. & Shrub & 2.6 & 2.6 & 4 & 2 & 3 & 2.84 & $S$ \\
\hline Rubus ellipticus Smith. & Shrub & 2.5 & & 3 & 3 & 3 & 2.87 & $\mathrm{~S}$ \\
\hline $\begin{array}{l}\begin{array}{l}\text { Ziziphus nummularia(Burm. f.) Wight } \\
\text { \&Arn. }\end{array} \\
\end{array}$ & Shrub & 2.6 & & & 2.4 & 3.75 & 2.91 & $S$ \\
\hline Withania somnifera $($ L. $)$ Dunal. & Shrub & & & & & 3 & 3 & $S$ \\
\hline Dodonaea vescosa $(\mathrm{L}.) \mathrm{Jacq}$. & Shrub & 3.75 & 2.6 & 3 & 3 & 3 & 3 & $S$ \\
\hline Mallotus philippensis(Lam.)Muess. & Shrub & 4 & 3.75 & 2 & 3.75 & 3 & 3.3 & $S$ \\
\hline Nerium indicumMill. & Shrub & 4 & & & & 2.6 & 3.3 & $\mathrm{~S}$ \\
\hline $\begin{array}{l}\text { Cedrus deodara(Roxb. ex D. Don), G. } \\
\text { Don. }\end{array}$ & Tree & 0.5 & 0.4 & 0.5 & 0.66 & 0.66 & 0.54 & $\mathrm{CE}$ \\
\hline $\begin{array}{c}\text { Pistacia integerrimaJ.L.Stewart. } \\
\text { Brandis. }\end{array}$ & Tree & 0.66 & 0.5 & 0.66 & 0.66 & 0.66 & 0.62 & $\mathrm{CE}$ \\
\hline Taxus wallichiana(Zucc.)Pilger. & Tree & 0.66 & & 1 & & & 0.83 & $\mathrm{CE}$ \\
\hline Ficus caricaForsk. & Tree & 0.66 & & 1 & 0.66 & 1.32 & 0.91 & $\mathrm{CE}$ \\
\hline Cornus macrophylla Wall. ex Roxb. & Tree & 1 & 1 & 1 & & & 1 & $\mathrm{CE}$ \\
\hline Bauhinia variegate $\mathrm{L}$. & Tree & 1.2 & 1.2 & 1 & 1 & 1 & 1 & $\mathrm{CE}$ \\
\hline Quercus dilatata Lindle. ex Royle. & Tree & 1.2 & & 1 & & & 1.1 & E \\
\hline Quercus leucotrichophora A. Camus. & Tree & 0.75 & & 1.5 & & & 1.12 & $\mathrm{E}$ \\
\hline Quercus floribundla Lindl. & Tree & 1 & & 1.3 & & & 1.15 & $\mathrm{E}$ \\
\hline Quercus incanaRoxb. & Tree & 1 & 0.6 & 1.3 & & 2 & 1.22 & $\mathrm{E}$ \\
\hline Populus alba $\mathrm{L}$. & Tree & 1.3 & 1.3 & & 1.3 & 1.3 & 1.3 & E \\
\hline Abies pindrow Royle. & Tree & 0.66 & & 2 & & & 1.33 & $\mathrm{E}$ \\
\hline Picea smithiana(Wall.) Boiss. & Tree & 0.66 & & 2 & & & 1.33 & $\mathrm{E}$ \\
\hline Butea monosperma (Lam.) O. Kuntz. & Tree & 1 & & 1.5 & 1.5 & 1.5 & 1.37 & E \\
\hline Grewia optiva Drummond .exBurret. & Tree & 1.6 & 0.75 & 1.42 & 1.6 & 1.66 & 1.4 & $\mathrm{E}$ \\
\hline Bombax ceiba $\mathrm{L}$. & Tree & 1 & 0.75 & 2 & 2.6 & 1 & 1.47 & $\mathrm{E}$ \\
\hline Celtis australis $\mathrm{L}$. & Tree & 1.2 & 1.2 & 1.5 & 2 & 1.5 & 1.48 & $\mathrm{E}$ \\
\hline Alnus nitida(Spach.) Endl. & Tree & 0.66 & 1 & 3 & 1.32 & & 1.49 & $\mathrm{E}$ \\
\hline Cydonia oblonga Miller. & Tree & 1.5 & & & & 1.5 & 1.5 & E \\
\hline Morus alba $\mathrm{L}$. & Tree & 1.5 & & 1.5 & 2 & 1 & 1.5 & $\mathrm{E}$ \\
\hline Phoenix dactylifera $\mathrm{L}$. & Tree & & & 1.5 & & & 1.5 & $\mathrm{E}$ \\
\hline Phoenix sylvestris(L.) Roxb. & Tree & & & & 1 & 2 & 1.5 & $\mathrm{E}$ \\
\hline
\end{tabular}




\begin{tabular}{|c|c|c|c|c|c|c|c|c|}
\hline \multirow{2}{*}{ Name of species } & \multirow{2}{*}{ Habit } & \multicolumn{7}{|c|}{ Tribe wise CS values } \\
\hline & & Basikhel & Nusratkhel & Akazai & Hassanzai & Medakhel & Mean & Categories \\
\hline Pinus wallichiana A. B. Jackson. & Tree & 1.5 & 1.5 & 1.5 & 1.5 & 1.5 & 1.5 & E \\
\hline Sapindus mukorossiGaertn. & Tree & 1.5 & & & & & 1.5 & $\mathrm{E}$ \\
\hline Acacia nilotica $\mathrm{L}$. & Tree & 1 & 1 & 2 & 2 & 2 & 1.6 & $\mathrm{~V}$ \\
\hline Ficus benghalensis $\mathrm{L}$. & Tree & 1.5 & 1.5 & 2 & 1.5 & 1.5 & 1.6 & $\mathrm{~V}$ \\
\hline Aesculus indica(Wall.exCamb.)Hk. & Tree & 1.33 & & 1.5 & & 2 & 1.61 & $\mathrm{~V}$ \\
\hline Albezzia procera(Roxb) Benth. & Tree & 1.5 & 1.5 & 3 & 1.5 & 1 & 1.7 & $\mathrm{~V}$ \\
\hline Pyrus communis $\mathrm{L}$. & Tree & 1.5 & & 2 & & & 1.75 & $\mathrm{~V}$ \\
\hline Ziziphus jujube Mill. & Tree & 1.6 & 1.6 & 2 & 1.2 & 2.6 & 1.8 & $\mathrm{~V}$ \\
\hline Albezzia lebbek(L) Benth. & Tree & 1.5 & & 2 & 2 & & 1.83 & $\mathrm{~V}$ \\
\hline Pyrus pashia Ham ex D. Don. & Tree & 2 & 1.33 & 2 & 2 & 2 & 1.86 & $\mathrm{~V}$ \\
\hline Ficus racemosa $\mathrm{L}$. & Tree & 2 & 2 & 2 & 2 & & 2 & $\mathrm{~V}$ \\
\hline Ilex dipyrena $\mathrm{Wall}$. & Tree & 2 & & & & & 2 & $\mathrm{~V}$ \\
\hline Cedrella serrata Royle. & Tree & 2 & & 1.5 & 3 & 2 & 2.12 & $\mathrm{R}$ \\
\hline Azadiracha indica $\mathrm{L}$. & Tree & 2.66 & 3.3 & 2 & 1 & 2 & 2.19 & $\mathrm{R}$ \\
\hline Delbergia sisso Roxb. & Tree & 2 & 2 & 2.6 & 2.6 & 2.5 & 2.34 & $\mathrm{R}$ \\
\hline Punica granatum $\mathrm{L}$. & Tree & 2.5 & 2.6 & 2 & 2 & 2.6 & 2.34 & $\mathrm{R}$ \\
\hline Diospyrus lotus L. & Tree & 2.5 & 2.3 & 2.5 & & & 2.43 & $\mathrm{R}$ \\
\hline Salix tetrasperma Roxb. & Tree & 1.5 & & & 2 & 4 & 2.5 & $\mathrm{R}$ \\
\hline Olea ferruginea Royle. & Tree & 3 & 2.4 & 2.5 & 3 & 3 & 2.78 & $\mathrm{~S}$ \\
\hline Pinus roxburghii Sargent. & Tree & 2 & 2 & 3.32 & 3.32 & 3.32 & 2.7 & $\mathrm{~S}$ \\
\hline Ficus palmate Forsk. & Tree & 3 & 3.5 & 2.5 & 3 & 2.5 & 2.9 & $S$ \\
\hline Ailanthus altissima(Mill.) Swingle. & Tree & 3 & 3 & 2 & 4 & & 3 & $\mathrm{~S}$ \\
\hline Robinia pseudoacacia $\mathrm{L}$. & Tree & 3 & 2.25 & 3 & 2.5 & 4.5 & 3 & $S$ \\
\hline Moras nigra $\mathrm{L}$. & Tree & 3 & 3 & 3.5 & 3 & 2.8 & 3 & $S$ \\
\hline Melia azedarach $\mathrm{L}$. & Tree & 3 & 3.5 & 3 & 3 & 3 & 3.1 & $S$ \\
\hline Acacia modesta Wall. & Tree & 3 & 3 & 3.75 & 3 & 3 & 3.15 & $S$ \\
\hline Juglans regia $\mathrm{L}$. & Tree & 3 & 4.2 & 3 & 3 & 3 & 3.24 & $S$ \\
\hline Prunus armeniaca $\mathrm{L}$. & Tree & 3 & 3.6 & & & & 3.3 & $S$ \\
\hline Euclaptus sp. & Tree & 2 & & & 6 & 4 & 4 & $S$ \\
\hline $\begin{array}{c}\text { Broussonetia papyrifera }(\mathrm{L} .) \mathrm{L}^{\prime} \text { Herit ex } \\
\text { Vent. }\end{array}$ & Tree & 4.5 & 4.5 & 5 & 4.98 & 4.98 & 4.79 & $S$ \\
\hline Platanus orientalis $\mathrm{L}$. & Tree & 5 & & & & & 5 & $S$ \\
\hline
\end{tabular}

NR: not reported, CS: conservation status, CE: critically endangered, E: endangered, V: vulnerable, R: rare, S: secure

\section{Conservation status of herbs}

Six species of herbs were found critically endangered (CE) showing CS values up to 1.0 These species are Podophyllum emodi (0.5), Gentiana kurroo (0.85), Dioscoreadeltoidea (0.88), Incarvellia emodi (0.96), Arisaema utile (1.0) and Picris hieraciodes (1.0) (Fig. 2). Thirteen species of herbs were mentioned as endangered ranging CS values from 1.1 to 1.5 . Vulnerable species were found 31 , rare 41 and secure 104 (Table 2).

\section{Conservation status of shrubs}

Among shrubs 9 species were found critically endangered. These were Skimmia laureola (0.48), Woodfordiafruticosa (0.78), Periploca aphylla (0.88), Hedra nepalensis (01), Nannorrhops ritchieana(01), Vitis jacquemontii (01), Viburnum grandiflorum (01) and Opuntia dillenii(01) (Fig. 3). Endangered species were recorded 4, vulnerable 15, rare 11 and secure 9 (Table 2). 


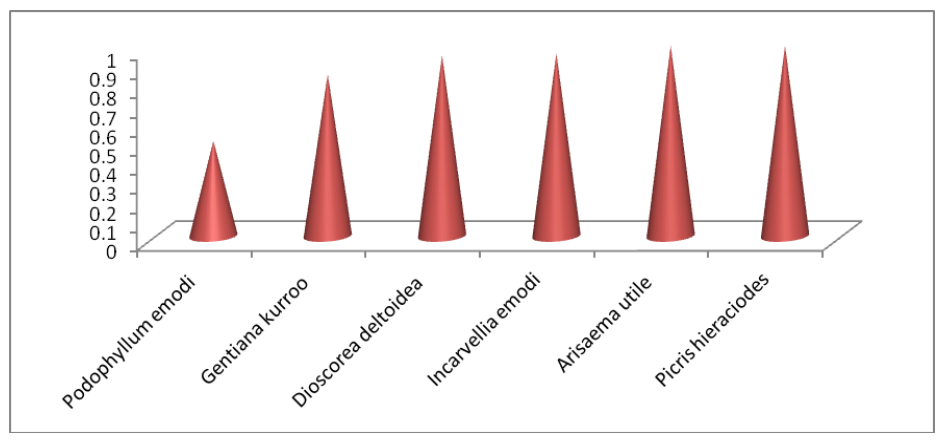

Figure 2. Critically endangered herbs of district Tor Ghar

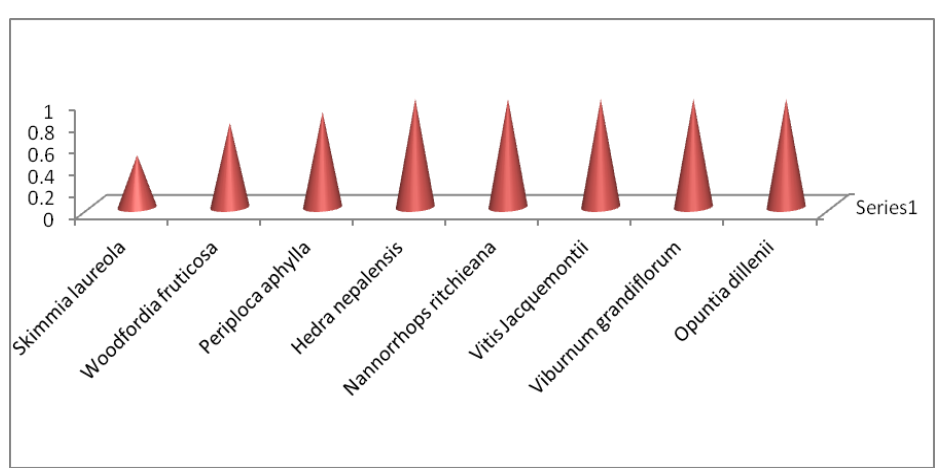

Figure 3. Critically endangered shrubs of district Tor Ghar

\section{Conservation status of trees}

Six trees species were declared critically endangered. These were Cedrus deodara (0.54), Pistacia integerrima (0.62), Taxus wallichiana (0.83), Ficus carica (0.91), Cornus macrophylla (1) and Bauhinia variegata (1) (Fig. 4). Eighteen species of trees were mentioned as endangered, 10 vulnerable, 6 rare and 13 secure (Table 2).

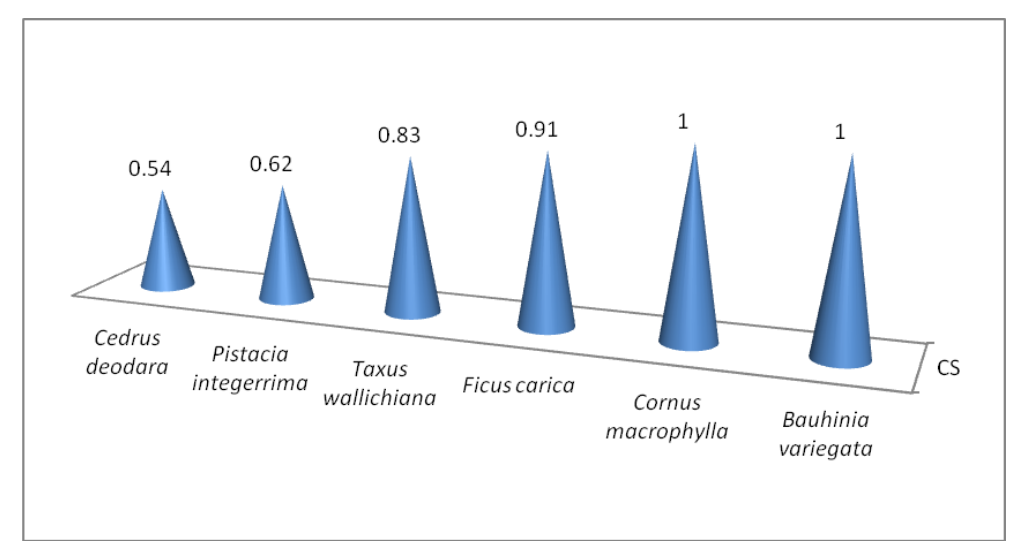

Figure 4. Critically endangered trees of district Tor Ghar

Herbs are found secure mostly in the region and least critically endangered whereas trees are more endangered and least critically endangered and rare. Shrubs are more vulnerable and least endangered (Fig. 5). 


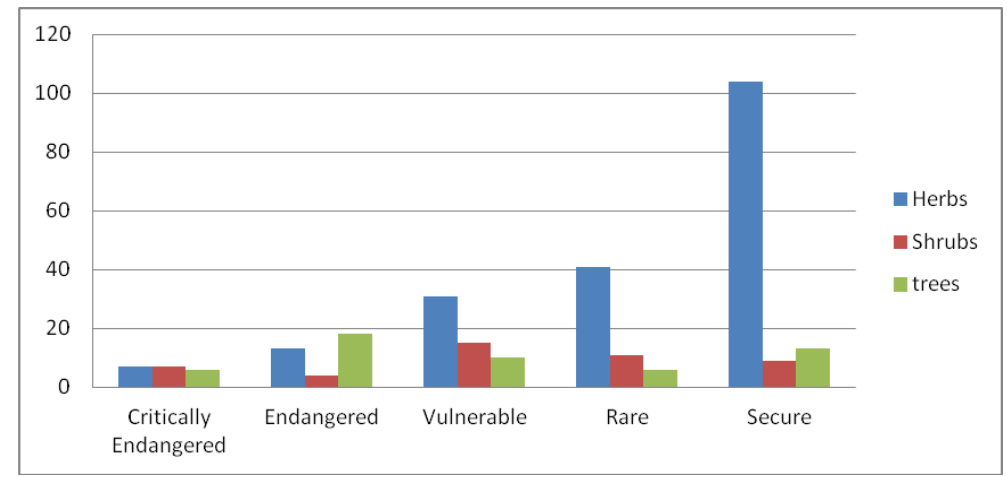

Figure 5. Number of species of all life forms and IUCN categories

Overall 20 species of all life forms were declared critically endangered, 35 endangered, 56 vulnerable, 58 rare and 126 secure (Fig. 6).

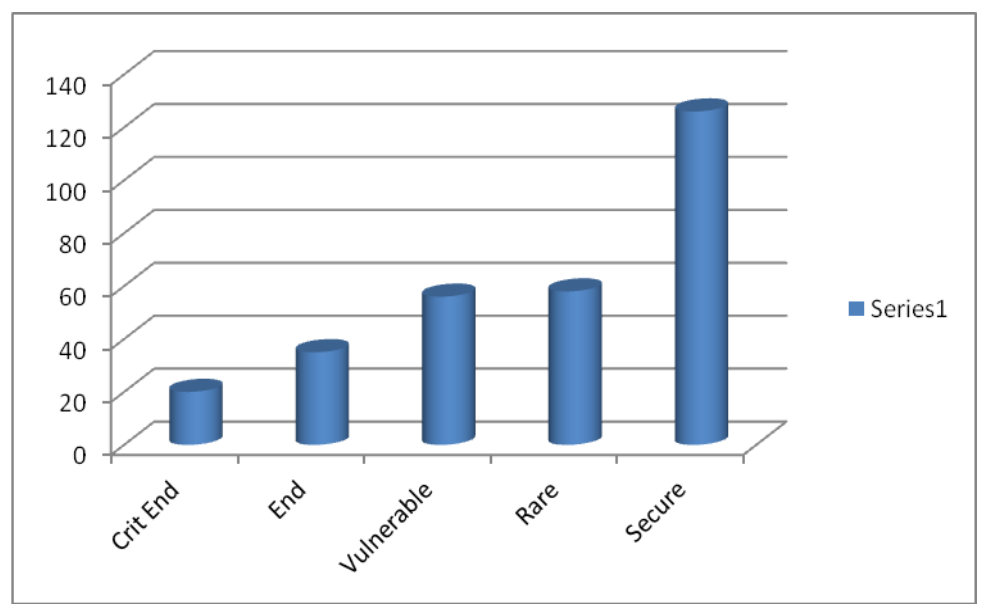

Figure 6. Total number of species assigned to IUCN categories

The percentage of threatened flora $38 \%$ (7\% critically endangered, $12 \%$ endangered, $19 \%$ vulnerable) 19\% near threatened (rare) was higher as compare to secure (least concerned) flora $43 \%$ (Fig. 7).

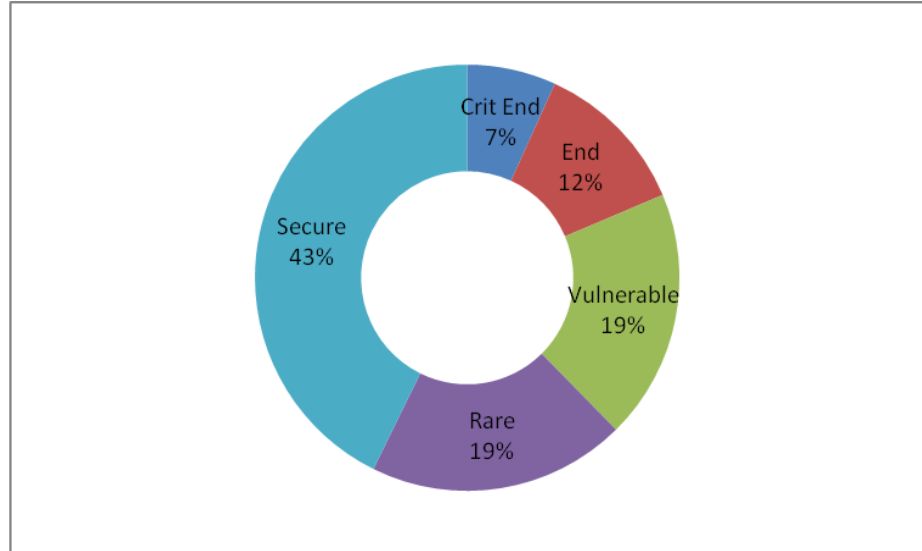

Figure 7. Percentage of threatened and secure species of Tor Ghar 


\section{Discussion}

The current investigation finds out the role of ethnoecological knowledge in evaluating the conservation status of culturally significant flora of district Tor Ghar. The United Nations Convention Environment and Development (UNCED, 1992) also highlighted the important roles that indigenous peoples play in both the conservation and sustainable use of natural resources while in turn maintaining biodiversity. In the present study local wisdom was explored and conservation status of 295 plants species in terms of IUCN categories was calculated on the basis of newly developed equation. The results depict $7 \%$ critically endangered flora, $12 \%$ endangered, $19 \%$ vulnerable and $19 \%$ near threatened (rare). The rest of the flora that is $43 \%$ found secure (least concerned). Such categorization was also found in a number of other ethnobotanical studies (Gilani et al., 2006; Afzal et al., 2009; Haq, 2012; Akhter et al., 2013; Bibi et al., 2014; Shah et al., 2015). Hamayun et al., 2006) also evaluated conservation status of 49 medicinal plant species and found 24 species threatened. Out of which 9 were found endangered, 7 vulnerable and 8 rare. The study also revealed that locals particularly plants collectors had useful information about plants occurrence, distribution and sites of their maximum availability. This information was cross checked through field surveys and visits. The field observations include, range extent and area of occupancy, exploitation level, availability, habitat alteration, conservation efforts, plant collection methods, part collected, invasive species and threats to plant biodiversity. On the basis of these observations, the species were then categorized according to IUCN categories as critically endangered, endangered, vulnerable, rare (near threatened) and secure (least concerned).

The results obtained by applying this equation show accuracy and found in accordance with the majority of results of other studies conducted in adjacent areas as well as different parts of Pakistan. According to this equation six species of herbs were found critically endangered (CE) showing CS values up to 1 . These species are Podophyllum emodi (0.5), Gentiana kurroo (0.85), Dioscorea deltoidea (0.88), Incarvellia emodi (0.96), Arisaema utile (1) and Picris hieraciodes (01). Thirteen species of herbs were mentioned as endangered ranging CS values from 1.1 to 1.5. Vulnerable species are 31 (1.6-2), rare 41 (2.1-2.5) and secure 104 (CS value above 2.5). Gilani et al. (2006) documented 21 ethnomedicinal herbs from AyubiaNational Park, district Abbottabad and found Podophyllum emodi and Viola canescensas vulnerable species due to overexploitation. While according to the findings of the present study Podophyllum emodi (0.5) is critically endangered but Viola canescens (2.7) is secure. Podophyllum emodi is under great harvesting pressure, showing minimum occurrence, availability for the last 10 years decreases and its regeneration potential could not keep pace with its overexploitation. Whereas Viola canescens shows the criteria of secure in this study. Afzal et al. (2009) studied that some plant species Colchicum luetum, Bergenia ciliata, Pimpinella stewartii, Valleriana jatamonsii, Viola serpens and Dioscorea deltoids etc. are rapidly depleting and may become locally extinct in near future. Some of these have also been reported and assessed in present investigation. Colchicum luetum (1.33), Bergenia ciliate (1.76) and Valleriana jatamonsii (1.92) were found vulnerable. While Dioscorea deltoids (.95) found critically endangered at local level.

Among shrubs 8 species were found critically endangered. These are Skimmia laureola (0.48), Woodfordia fruticosa (0.78), Hedra nepalensis (1.0), Nannorrhops ritchieana (1.0), Vitis jacquemontii (1.0), Periploca aphylla (01), Opuntia dillenii (01) 
and Viburnum grandiflorum (1). Endangered species are 4, vulnerable 15, rare 11 and secure 9 (Table 2).

Six trees species were declared critically endangered. These are Cedrus deodara (0.54), Pistacia integerrima (0.62), Taxus wallichiana (0.83), Ficus carica (0.91), Cornus macrophylla (1) and Bauhinia variegate (1.0). Eighteen species of trees were declared as endangered, 10 vulnerable, 6 rare and 13 secure (Table 2).

Similarly Haq (2012) also conducted ethnoconservation survey of 12 threatened species from district Battagram (adjacent district to Tor Ghar). These species were Acer caesium, Betula utilis, Cedrus deodara, Opuntia dilleni, Paeonia emodi, Pistacia integerrima, Populus alba, Quercus glauca, Skimmia laureola, Taxus wallichiana, Ulmus wallichiana and Viscum album. Their conservation status was assessed through direct field observation and the information gathered by locals. The present research work also declared 6 plants Cedrus deodara, Opuntia dilleni, Pistacia integerrima, Populus alba, Skimmia laureola and Taxus wallichiana as critically endangered species of district Tor Ghar.

Shah et al. (2012) explored Olea ferruginea along with mixed tree species of Monotheca buxifolia and Acacia modesta as dominant ethnoflora of sacred jungles of Kurd sharif and Sho of district Karak showing a traditional way of conserving plant biodiversity. Similar findings of this research show Olea ferruginea (2.78) and Acacia modesta (3.15) as secure species of the study area. Olea ferruginea is protected in graveyards and considered one of the most important sacred plant species of Graveyards and is not cut for any purpose. Olea ferrugineais also protected by locals from fire by making small circular stony walls around trees (Shah et al., 2015)

Ahmad et al. (2012) found 5 plants species critically endangered, 6 endangered, 10 vulnerable, 11 secure and 6 rarely distributed in Sharda, Neelam valley Azad Jammu and Kashmir. This study further revealed the over exploited species are Aconitum heterophyllum, Geranium wallichianum, Ajuga bracteosa, Traxicum officinale, Quercus incana, Berberis lycium, viola canescens and Dyosporus lotus. Majority of them are at the verge of local extinction due to extensive harvesting pressure. The CS values index also shows Geranium wallichianum (1.56), Ajuga bracteosa (02) and Berberis lyceum (02) as vulnerable species, whereas Traxicum officinale (2.13) and Dyosporus lotus (2.4) as rare species. Whereas Quercus incana (1.22) as endangered species and viola canescens (2.7) as secure species. The result of viola canescens (2.7) as secure species is only different. It may be due to its habitat adjustment or reduced overexploitation. Therefore, viola canescens (2.7) is not considered the threatened species of the district. The field observations also show its moderate occurrence.

In Chakesar valley district Shangala, a plant resource assessment project was carried out ethnobotanically to explore conservation status of some important medicinal plants. Conservation of 127 plant species was evaluated through IUCN criteria. Among these species 47 were endangered (E), 32 vulnerable (VU), 36 rare (R) and 12 species were infrequent (IF). Some of the important endangered species of the region are Abies pindrow, Acer oblongum, Aesculus indica, Alnus nitida, Berberis kunawarensis, Celtis australis, Desmodium elegans, Hedrane palensis, Juglan regia, Olea ferruginea, Paeonia emodi, Picea smithiana, Pinus gerardiana, Pisticia integrima, Quercus semicarfifolia, Vibernum grandiflorum and Woodfordia fruticosa (Shah and Hussain, 2012). When the results of this study were compared with the results of present research, majority of the species showed similarity in results and however, a few species showed different conservation stata e.g. Abies pindrow, Alnus nitida, Celtis australis 
and Picea smithiana were declared as endangered species while Hedrane palensis, Vibernum grandiflorum and Woodfordia fruticosa as critically endangered species of the research area. Aesculus indica was mentioned as vulnerable species of the district. In these results category of threatening is different. However, Juglan regia and Olea ferruginea showed different results being culturally significant species. These are well protected by local culture and therefore, attained the secure status. Juglan regia is the most popular wild edible plant of the study area. This tree species provides a number of services to the locals in the form of furniture wood, cosmetics and tasteful dry fruit having prolonged storage life. This important species is facing overexploitation pressure but due to cultivation and protection by locals showing secure status in the district Tor Ghar (Shah et al., 2015).

Akhtar et al. (2013) assessed ethnobotanically that Aconitum violaceum, Colchicum luteum and Jasminum humile as vulnerable due to over exploitation. According to the present study Colchicum luteum (1.33) is endangered and Jasminum humile (2.33) is rare. However, Aconitum violaceum was not reported by any tribe in the current study.

Bibi et al. (2014) pointed out the highest under pressure species Caralluma tuberculata, Citrullus colocynthis, Malva neglecta and Mentha longifolia in district Mastung of Baluchistan. Caralluma tuberculata (1.32) and Citrullus colocynthis (1.1) were also declared as endangered species, whereas Malva neglecta (3) and Mentha longifolia (4.49) as the most secured species of the area showing highest values of CS. Malva neglecta is an agricultural land associated plant species and is found abundantly in the study area. Similarly, Mentha longifolia was found secure and grows excessively near aquatic habitats of Tor Ghar.

Mussarat et al. (2014) indicated Morus alba and Dalbergia sissoo under great overexploitation pressure and threatened species in the area. While in the present exploration Morus alba (1.5) is endangered and Dalbergia sissoo (2.3) is rare. Dalbergia sissoo is under great overexploitation pressure especially for furniture wood. Morus alba is also depleting alarmingly in the region. No conservatory measures are taken to secure Morus alba in Tor Ghar.

Amjad et al. (2015) indicated Ajuga bracteosa, Mallotus philippensis, Micromeriabiflora, Butea monospermaand Zanthoxylum armatum as critically endangered in Kotli, Azad Jammu and Kashmir. The present research work show Ajuga bracteosa (2) as vulnerable, Mallotus philippensis (3.3) as secure, Butea monosperma (1.37) as endangered species and Zanthoxylum armatum (2.4) as rare species.

In the current study Taxus wallichiana (0.83) was found critically endangered while Pinus wallichiana (1.5) and Abies pindrow (1.33) were found endangered. The study of Adnan et al. (2006) also mentioned these species as rapidly decreasing species in Miandam vally of district Swat.

Cedrus deodara in this study showing (0.54) CS value and declared critically endangered. Sheikh et al. (2002) also pointed out that Cedrus deodara had completely eradicated from Naltar valley, northern western Karakorum range.

When the results of present study were matched with IUCN red list criteria at regional level two species were found critically endangered (CE) in the study area. These species were Cedrus deodara (0.54) and Gentiana kurroo (0.85). Two species were found Endangered/Threatened (EN) in the region i.e., Picea smithiana (1.33) and Viburnum cotinifolium (1.13). While Geranium wallichianum (1.56) and Salvia moorcroftiana (1.87) were mentioned endangered in IUCN criteria but vulnerable species at district Tor Ghar level. Bergenia ciliata (1.76) is vulnerable in IUCN list as 
well as in the present research. Pinus wallichiana (1.5) was found endangered in this study but vulnerable in IUCN red list. Viola canescens (2.7) is considered as secure locally but vulnerable regionally. This species showed quite different conservation status from the published literature and IUCN red list indicating its conservation status secured locally.

\section{Conclusion}

Local ecological knowledge has been proved a successful tool for evaluating conservation stata of flora of the study site. This empirical study revealed that a higher proportion $(57 \%)$ of flora is undergoing various degrees of threatening at local level that is $7 \%$ critically endangered, $12 \%$ endangered, $19 \%$ vulnerable and $19 \%$ rare in terms of IUCN categories. However, forty three percent flora of the district is secure. The findings of the present study indicating that flora of Tor Ghar is under high anthropogenic pressure. This is first ever attempt of quantifying the local wisdom regarding conservation status of the plants. Local perception of the flora also reflects its conservation status which may be considered in futures researches as base line study. The above discussion revealed that the mathematical relation designed during the study has been proved useful tool for evaluating ethno-conservation of local flora. The equation can be generalized and applied to any floristic area of the world. Conservation researchers should quantify the local wisdom while applying IUCN criteria to any floristic territory especially where anthropogenic disturbances are at alarming rate. This will reinforce the results and more clarify the picture of conservation status of plant biodiversity of a region.

Acknowledgements. Indigenous tribes of district Tor Ghar are highly acknowledged for their permission of gathering data and cooperation and guidance during field trips.

Conflict of interests. The authors declare that they have no competing interests.

\section{REFERENCES}

[1] Adams, W. M., Hutton. J. (2007): People, parks and poverty: political ecology and biodiversity conservation. - Conservation and Society 5: 147-183.

[2] Adams, W. M., Aveling, R., Brockington, D., Dickson, B., Elliott, J., Hutton, Roe, D., Vira, B., Wolmer, W. (2004): Biodiversity conservation and the eradication of poverty. Science 306(5699): 1146-1149. http://dx.doi.org/10.1126/science.1097920.

[3] Adnan, S. M., Khan, A. A., Latif, A., Shinwari. Z. K. (2006): Threats to the sustainability of ethno-medicinal uses in northern Pakistan. A case study of Miandam valley, district Swat, NWFP province, Pakistan. - Lyonia 11(2): 19-100.

[4] Afzal, S., Afzal, N., Awan, M. R., Khan, T. S., Gilani, A., Khanum, R., Tariq, S. (2009): Ethno-botanical studies from Northern Pakistan. - Journal of Ayub Medical College Abbottabad 21(1): 52-56.

[5] Ahmad, K. S., Qureshi, R., Hameed, M., Ahmad, M. F., Nawaz, T. (2012): Conservation assessment and medicinal importance of some plants resources from Sharda, Neelumvalley, Azad Jammu and Kashmir, Pakistan. - International Journal of Agriculture and Biological Sciences 14: 997-1000. 
[6] Akhtar, N., Rashid, A., Murad, W. E., Bergmeier, E. (2013): Diversity and use of ethnomedicinal plants in the region of Swat, North Pakistan. - J. Ethnobio. \& Ethnmed. DOI: 10.1186/1746-4269-9-25.

[7] Amjad, M. S., Arshad, M. R., Quershi, M. (2015): Ethnomedicinal inventory and folk uses of indigenous plants from Pir Nasoora National Park, Azad Jammu and Kashmir. Asian Pacific J. Trop. Biomed. 5(3): 234-241. 9: 25.

[8] Andrews, G., Daylight, C., Hunt, J. (2006): Aboriginal cultural heritage landscape mapping of coastal NSW. - Prepared for the Comprehensive Coastal Assessment by the NSW Department of Natural Resources, Sydney, NSW.

[9] Anonymous (1992): CBD (Convention on Biological Diversity). - The Convention on Biological Diversity, Rio de Janeiro.

[10] Aswani, S., Lemahieu, A., Sauer, W. H. H. (2018): Global trends of local ecological knowledge and future implications. - PLoS One 13(4): e0195440. https://doi.org/10.1371/journal.pone.0195440.

[11] Berkes, F. (2007): Community-based conservation in a globalized world. - Proceedings of the National Academy of Sciences of the United States of America 104(39): 1518815193.

[12] Berkes, F., Colding, J., Folke, C. (2000): Rediscovery of traditional ecological knowledge as adaptive management. - Ecol Appl. Ecological Society of America 10: 1251-1262.

[13] Bibi, T., Ahmad, M., Tareen, R. B., Tareen, N. M., Jabeen, R., Rehman, S. U., Sultana, S., Zafar, M., Yasee, G. (2014): Ethnobotany of medicinal plants in district Mastung of Balochistan Province-Pakistan. - J. Ethnopharmacol. 157: 79-89.

[14] Boom, B. M. (1987): Ethnobotany of the Chacobo Indians, Beni, Bolivia. - Adv. in Econ. Bot. 1-68.

[15] Bridgewater, P. B. (2002): Biosphere reserves: special places for people and nature. Environ. Sci. \& Policy 5: 9-12.

[16] Broennimann, O., Vittoz, P., Moser, D., Guisan, A. (2005): Rarity types among plant species with high conservation priority in Switzerland. - Bot. Helv. 115: 95-108.

[17] Calvo, L.M. (2003): EI humano y la gestion de la biodiversidad biologica: Problemas, potencialidades y tendencies. - In: Ibish, P. L., Merida, G. (eds.) Biodiversidad: Lariqueza de Bolivia. Estado de conocimiento y Conservacion. Ministerio de Desarrollo Sostenible. Editorial FAN, Santa Cruz de la Sierra, Bolivia.

[18] Cotton, C. M. (1996): Ethnobotany. Principles and Applications. - John Wiley and Sons, New York.

[19] Domínguez Lozano, F., Moreno Saiz, J. C., SainzOllero, H. (2003): Rarity and threat relationships in the conservation planning of Iberian flora. - Biodivers. \& Conserv. 12: 1861-1882.

[20] Gilani, S. A., Quershi, R. A., Gilani, S. J. (2006): Indigenous uses of some important ethnomedicinal herbs of Ayubia National Park, Abbottabad, Pakistan. - Ethnobotany Leaflets 10: 285-293.

[21] Grimble, R., Laidlaw, M. (2002): Biodiversity management and local livelihoods: Rio plus 10. - ODI Natural Resource Perspectives Papers 73: 1-4.

[22] Hamayun, M., Khan, S. A., Sohn, E. Y., Lee, I. J. (2006): Folk medicinal knowledge and conservation status of some economically valued medicinal plants of district Swat, Pakistan. - Lyonia 11(2): 101-113.

[23] Haq, F. (2012): The critically endangered flora and fauna of district Battagram, Pakistan. - Advances in life Sciences 2(4): 118-123.

[24] Jules, P. (2008): How Do Biodiversity and Culture Intersect? Sustaining Cultural and Biological Diversity in a Rapidly Changing World: Lessons for Global Policy, 2-5 April 2008. - American Museum of Natural History's Center for Biodiversity and Conservation, IUCN-The World Conservation Union/Theme on Culture and Conservation, and Terralingua, pp. 1-17. 
[25] Larid, S. A. (1999): Forests, Culture and Conservation. - In Posey, D. A. (ed.) Cultural and Spiritual Values of Biodiversity. - UNEP and Intermediate Technology Publications: London, pp. 345-396.

[26] McNeely, J. A. (2000): Cultural Factors in Conserving Biodiversity. - In Wilkes, A., Tillman, H., Salas, M., Grinter, T., Shaoting, Y. (eds.) Links between Cultures and Biodiversity. Proceedings of the Cultures and Biodiversity Congress. Yunnan Science and Technology Press, Yunnan, P. R. China, pp. 128-142.

[27] Meijaard, E., Mengersen, K., Buchori, D., Nurcahyo, A., Ancrenaz, M., Wich, S. (2011): Why don't we ask? A complementary method for assessing the status of great apes. PLoS One 6(3): e18008. https://doi.org/10.1371/journal.pone.0018008.

[28] Mussarat, S., Salam, N. M. A., Tariq, A., Wazir, S. M., Ullah, R., Adnan, M. (2014): Use of ethnomedicinal plants by the people living around Indus River. Evid. based complement. - Alternat. Med. 2014: 2.

[29] Negi, C. S. (2010): Traditional culture and biodiversity conservation: examples from Uttarakhand, Central Himalaya. - Mounain Research \& Development30: 259-265.

[30] Phillips, O., Gentry, A. H. (1993b): The useful plants of Tambopata, Peru: 11. Additional hypothesis testing in quantitative ethnobotany. - Economic Botany 47: 33-43.

[31] Posey, D. A. (1999): Cultural and Spiritual Values of Biodiversity. A Complementary Contribution to the Global Biodiversity Assessment. - In Posey, D. A. (ed.) Cultural and Spiritual Values of Biodiversity. UNEP and Intermediate Technology Publications, London, pp. 1-19.

[32] Posey, D. A., Overal, W. L., Goeldi, M. P. E. (1990): Ethnobiology: implications and applications. Proceedings of the First International Congress of Ethnobiology, Belém, 1988.

[33] Prance, G. T., Balee, W., Boom, B. M., Carneiro, R. L. (1987): Quantitative ethnobotany and the case for conservation in Amazonia. - Conservation Biology 1: 296-310.

[34] Rajeswar, J. (2001): Conservation ethics versus development: How to obviate the dichotomy. - Sustainable Development 9: 16-23.

[35] Schwartzman, S., Nepstad, D., Moreira, A. (2000): Arguing tropical forest conservation: people versus Parks. - Conservation Biology 14: 1370-1374.

[36] Shah, A. H., Khan, S. M., Shah, A. H., Mehmood, A., Rehman, I. U., Ahmad, H. (2015): Cultural uses of plants among Basikhel tribe of district Tor Ghar, Khyberpakhtunkhwa, Pakistan. - Pakistan Journal of Botany 47(SI): 17-31.

[37] Shah, A. S., Hussain, S., Din, N. U., Bhatti, K. H., Khan, A., Marwat, S. K., Zafar, M., Ahmad, M. (2012): Sacred jungles. A traditional way of conserving endangered ecosystems and biodiversity in semi- tribal area Kurd Sharif \& Sho (District Karak, Khyber Pakhtunkhawa) Pakistan. - Science Technology and Development 31(4): 312326.

[38] Shah, M., Hussain, F. (2012): Conservation assessment of plant resources of Chakaser valley, District Shangla, KPK, Pakistan. - Pakistan Journal of Botany 44(SI): 179-186.

[39] Sheikh, K., Ahmad, T., Khan, M. A. (2002): Use, exploitation and prospects for conservation: people and plant biodiversity of Naltar Valley, northern western Karakorum, Pakistan. - Biodiversity and Conservation 11(4): 715-742.

[40] United Nations (1992): United Nations Framework Convention on Climate Change. United Nations, New York.

[41] Von Glasenapp, M., Thornton, T. F. (2011): Traditional ecological knowledge of Swiss alpine farmers and their resilience to socioecological change. - Human Ecology 39: 769781.

[42] Wells, M. P., McShane, T. O. (2004): Integrating protected area management with local needs and aspirations. - AMBIO: A Journal of the Human Environment 33: 513-519.

[43] Wiersum, K. F., Shackleton, C. M. (2005): Rural Dynamics and Biodiversity Conservation in Southern Africa. - In Ros-Tonen, M. A. F., Dietz, T. (eds.) African 
Forests between Nature and Livelihood Resources: Interdisciplinary Studies in Conservation and Forest Management. Edwin Mellen Press, UK.

[44] Zent, S. (2001): Acculturation and Ethnobotanical Knowledge Loss among the Piaroa of Venezuela: Demonstration of a Quantitative Method for the Empirical Study of Traditional Environmental Knowledge Change. - In: Maffi, L. (ed.) On Biocultural Diversity: Linking Language, Knowledge, and the Environment. Smithsonian Institution Press, Washington, DC, pp. 190-211. 